\title{
File Transmission and Download System Based on P2P
}

\author{
Liu Hongqing ${ }^{1}$, Liu Yan ${ }^{2}$ \\ ${ }^{1}$ Hunan Vocational College of Modern Logistics, Changsha, Hunan, 410131, China \\ ${ }^{2}$ Hunan Mechanical \& Electrical Polytechnic, Changsha, Hunan,410151, China
}

Keywords: P2P, upload, download, file system, distributed

\begin{abstract}
With the development of modern information technology, P2P technology has become a hot topic in computer network technology. Compared with the C/S mode, the P2P mode reduces the load of the server and reduces the network congestion; changes the client's passive role in the C/S structure, making the client a node with both the server and the client functions in the P2P network. JXTA (Juxtapose abbreviation, side by side) is mainly used to provide basic services for P2P programs. The technology is committed to create a universal platform to build specific equalities and distributed services and applications in a simple and effective way.
\end{abstract}

\section{Introduction}

FTP is the most widely used file transfer protocol on file in the Internet. As a general protocol, FTP allows arbitrary files and allows files to have ownership and access rights. Because of the details of the independent computer system, FTP is suitable for heterogeneous systems. It can transfer files between any computers.

Like other traditional networks, FTP uses client / server mode. When a large amount of data is distributed through the network to the dispersed users, the system uploads the published files to the server, and then notifies the users to download files from the center server. The processing capacity and transmission speed of servers are the bottlenecks affecting the speed of file distribution. As the number of users increases, the load pressure of the server becomes larger, and the download speed for each user will be reduced and the downloading time is longer, so many servers will limit the number of users and download speed. It brings a lot of inconvenience to the user.

\section{File transmission and downloading based on P2P}

$\mathrm{P} 2 \mathrm{P}$ is a technology used to exchange data or services directly between different PC users without relay devices. It allows users in the network to directly connect to other users' computers to exchange files. Under certain information security, people can share files on the hard disk via P2P instead of the same connection as in the past. Go to the server to browse and download. Because eliminating intermediate links, P2P technology makes information communication on the Internet easier and more direct.

The biggest difference between P2P and the traditional FTP is that users do not search and download resources from other web servers, but download them directly from any online peer. Of course, the servers of other websites can be considered as a peer, and the personal computer and the server can be really equal. The need for file exchange naturally extends to information exchange.

\section{JXTA brief introduction}

JXTA is a collaborative research project for P2P computing. The "JXTA project" (or JXTA) represents the "Juxtapose project" for this reason: Although client / server and network based computing is a very important standard today, it is also very urgent for a variety of applications oriented P2P standards. These applications have some common features.

(1) they are spontaneously spontaneous.

(2) when supported architectures, including many distributed service providers, they are more 
efficient than a separate centralized server.

(3) users of such applications can be all service providers or consumers that are abstracted by applications. They often play two roles at the same time.

(4) users of this application are constantly changing, so they may not be accessed at any particular point in time.

(5) users of the application may use any device at any time and place.

The traditional client / server computing model is hard to achieve such demand applications. It is based on a set of peers that cooperate and operate independently, and can communicate with each other in any network infrastructure. They will run better.

JXTA defines a set of public protocols for P2P computing. Each protocol is easy to implement and integrate into the existing system. It is also the group of protocols that define JXTA. JXTA is not API. Although standard language binding can be applied to JXTA protocol, binding is not mandatory. Application developers and hardware vendors can provide their own language binding. The new language develops and provides their own binding to the JXTA protocol; the new platform can be developed to achieve JXTA protocol in a completely new way. JXTA is independent of language and platform. Further speaking, JXTA is network independent: JXTA protocol can be transmitted by TCP/IP, Bluetooth, home network and so on. Peers in different networks can easily communicate with standard JXTA protocol. JXTA is a set of protocols that are independent of language and network. It makes it possible to develop applications and services on changing collaborative computing devices.

\section{JXTA architecture}

The JXTA is made up of three layers, as shown in Figure 1. The first layer is the core layer of the JXTA, which contains the core functions required by the service; the second layer is the service layer, which provides the interface to access the JXTA protocol; the third layer is the application layer, which uses services to access the JXTA network and the functions provided by the JXTA.

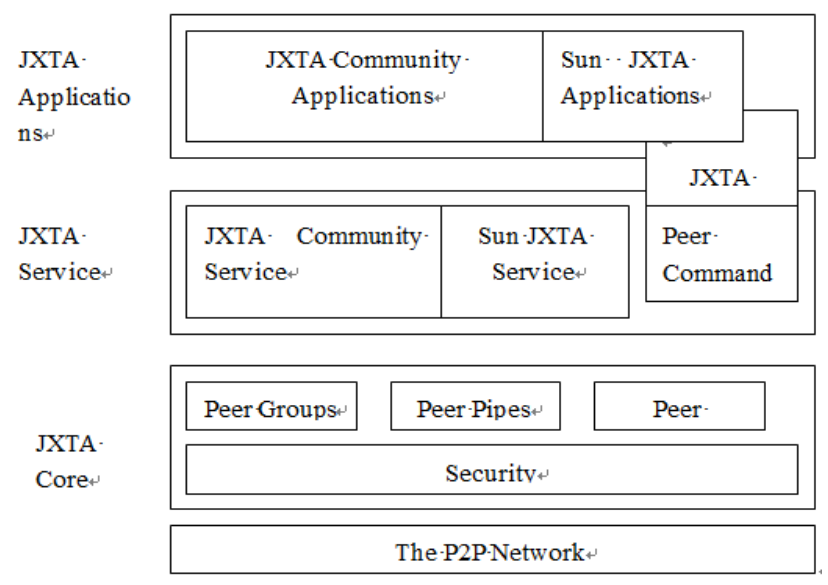

Figure 1 JXTA architecture diagram

(1) the platform (platform Layer), which is the bottom of the JXTA system and the core layer (JXTA Core), encapsulates the most basic things, including the peer, the peer group, the peer monitoring and the related security mechanisms. It is mainly responsible for the creation of peers, the communication management of similar routes, and other underlying transactions. This layer is designed to be refined and accurate, not only to maintain good interoperability in different P2P applications, but also to provide the maximum space for developers to facilitate the birth of new applications.

(2) peer group (Peer Groups): peer groups organize peers into a set, name them, and set corresponding mechanisms for creating, deleting, member management, publishing, and searching for other peer groups and peers, communication, security, content sharing and other basic functions.

Pipeline (Peer Pipes): pipeline provides communication channels between peers. The 
information transmitted in the pipeline is in the form of XML. In a sense, it does not depend on specific protocols. It also allows a certain degree of security, information integrity and personal privacy.

(3) peer monitoring (Peer Monitoring): the ability to control the behavior and activity of a peer group in a peer group can be used to implement peer management functions, including access control, priority setting, traffic calculation, and generation width equalization.

The core layer supports anonymous or registered users, content encryption or plaintext strategy, so that developers can not directly face the choice of special strategies. Policy selection is established at service level or application level. When necessary, policy selection can also be implemented in service layer or application layer. The core layer, as its name shows, is the foundation of JXTA platform. The service layer and application layer in JXTA application are built on this level.

(4) the service layer (Services Layer) is built on the core of the JXTA, extending the capabilities of the core layer and providing some operations that are not necessary for the P2P network, but these operations may be very common in the P2P environment. This layer provides convenience for the development of P2P applications, which include lookup and indexing, creation of directories, storage systems, file sharing, distributed file systems, resource centralization, protocol conversion, identity authentication and public key infrastructure (PKI, Public Key Tnfrastructure) services. This layer is the foundation of the application layer.

(5) the application layer (Applications Layer) is built on the service layer, including the implementation of integrated applications, such as $\mathrm{P} 2 \mathrm{P}$ instant messaging, file and resource sharing, entertainment content management and distribution, P2P e-mail systems, distributed auction systems, and many other applications.

The boundaries of services and applications are not very strict. The application of a user may be regarded as service by another user. The design of the whole system is modular, allowing developers to pick up and select the most suitable services and applications for them.

\section{Summary}

First, the definition, structure and mode of P2P, comparison with $\mathrm{C} / \mathrm{S}$ mode, development process and status quo, and development in China are introduced in detail. Then the definition, architecture and synergy of the key technology of this subject, JXTA, are analyzed in detail, and the JXTA CMS model used in this topic is further elaborated. Finally, a brief summary of the design is made. The system finally achieves the functions of file transmission, download and search. P2P technology has great advantages in communication network. The network constructed by P2P technology has become an important development direction of communication network.

\section{References}

[1] Jennifer W. Chan, Yingyue Zhang, and Kathryn E. Uhrich, Amphiphilic Macromolecule Self-Assembled Monolayers Suppress Smooth Muscle Cell Proliferation, Bioconjugate Chemistry, 2015, 26(7), 1359-1369.

[2] Jennifer W. Chan, Yingyue Zhang, and Kathryn E. Uhrich, Amphiphilic Macromolecule Self-Assembled Monolayers Suppress Smooth Muscle Cell Proliferation, Bioconjugate Chemistry, 2015, 26(7), 1359-1369.

[3] Kurup, P.; Sullivan, C.; Hannagan, R.; Yu, S.; Azimi, H.; Robertson, S.; Ryan, D.; Nagarajan, R.; Ponrathnam, T.; Howe, G. A Review of Technologies for Characterization of Heavy Metal Contaminants. Indian Geotech J 2017, 47 (4), 421-436.

[4] Ghebrebrhan, M.; Aranda, F.; Walsh, G.; Ziegler, D.; Giardini, S.; Carlson, J.; Kimball, B.; Steeves, D.; Xia, Z.; Yu, S.; et al. Textile Frequency Selective Surface. IEEE Microwave and Wireless Components Letters 2017, 27 (11), 989-991.

[5] Malarkodi, M.P., Arunkumar, N., Venkataraman, V. Gabor wavelet based approach for face recognition (2013) International Journal of Applied Engineering Research, 8 (15), pp. 1831-1840. 